\title{
The progress of, challenges faced by, and future of rare disease patient organizations in China
}

\author{
Rufang Huang ${ }^{1}$, Yunshu Wei ${ }^{1}$ Jiahao $\mathrm{Hu}^{2}$, Fanxin Kong ${ }^{3}$, Jiangjiang $\mathrm{He}^{4}$, Yan Yang ${ }^{4}$, \\ Mi Tang ${ }^{4}$, Chunlin Jin ${ }^{4}$, Qi Kang, ${ }^{4, *}$ \\ ${ }^{1}$ Chinese Organization for Rare Disorders, Shanghai, China; \\ ${ }^{2}$ Department of Learning, Informatics, Management and Ethics, Karolinska Institutet, Solna, Sweden; \\ ${ }^{3}$ School of International Pharmaceutical Business, China Pharmaceutical University, Nanjing, China; \\ ${ }^{4}$ Department of Health Policy Research, Shanghai Health Development Research center, Shanghai Medical Information Center, \\ Shanghai, China.
}

\begin{abstract}
Summary In addition to difficulties with treatment and expenses, patients with rare diseases in China greatly lack social support. In around 2000, Chinese patients with rare diseases and their families began to organize a series of charitable activities such as medical aid, public education, and policy advocacy. After nearly 20 years, organizations for Chinese patients with rare diseases have progressed. Many problems still remain, including a relatively small number of organizations, a low level of specialization, a lack of stability, limited social influence, and limited access to social resources. In order to spur the development of Chinese rare disease patient organizations, public education needs to be enhanced, policy support is needed, teams need to be created, and communication and cooperation need to be enhanced.
\end{abstract}

Keywords: Rare disease, patient organization, China

\section{Introduction}

In May 2018, five Chinese national agencies jointly published China's First National List of Rare Diseases, which included 121 diseases (1). This is the first time that rare diseases have been clearly delineated by the Chinese Government at the national level (2). Several policies on rare diseases in China have been promulgated since the list was issued. As the government and society pay greater attention to rare diseases, industry has begun to turn its attention to patients with rare diseases. However, Chinese patients with rare disease still face many difficulties, including the low level of diagnosis and treatment and limited access to orphan drugs (3). In addition to treatment and economic difficulties, one serious problem - lack of social support - is often overlooked $(4,5)$.

\footnotetext{
*Address correspondence to:

Dr. Qi Kang, Shanghai Health Development Research Center, Shanghai Medical Information Center, No.1477 Beijing Road West, Shanghai 200040, China.

E-mail: kangqi@shdrc.org
}

2. Rare disease patient organizations and their function

A patient organization is a non-profit organization created by patients with a certain disease or family members to represent and advocate for the overall interests of patients, to integrate resources from different stakeholders, and to provide services to the patients or their family (6). Rare disease patient organizations need to be established to provide social support due to the low prevalence of those diseases, the small number of patients, the lack of medical care, the psychological pressure on patients and their families, and the intense sense of loneliness.

Rare disease patient organizations originated in the US since the 1980s and then emerged in Europe and Japan (7). In addition to the functions mentioned earlier, rare disease patient organizations play an increasingly important role in research on rare diseases and orphan drugs, including enrollment in registries, helping to fund research, and participating in clinical trials $(8)$.

3. The development of rare disease patient organizations in China 
The development of rare disease patient organizations in China can be roughly divided into three stages:

Early Days (2000-2005) In around 2000, Chinese patients with rare diseases and their families began to assemble and organize a series of charitable activities such as medical aid, public education, and policy advocacy. Chinese patients with hemophilia have taken the lead in establishing a platform for communication and mutual assistance through the Internet, thus opening the way for the development of rare disease patient organization in China.

On the Rise (2006-2010) The Chongqing Hemophilia Rehabilitation Association was established in 2006, becoming the first patient organization registered in civil affairs bureau of China (7). In 2008, the China-Dolls Care and Support Association (the predecessor of the China-Dolls Center for Rare Disorders, or CCRD) was created by patients with osteogenesis imperfecta. The first special fund for patients with rare diseases in China, the Dolls Care and Support Fund, was set up by the CCRD under the China Social Welfare Foundation (9).

Further Development (since 2011) In 2011, the CCRD held a "Meeting on Capacity-building for Rare Disease Patient Organizations". The meeting focused on how to establish a standardized NGO and the creation of a platform for a rare disease network. Representatives from patient organizations representing 18 rare diseases jointly launched the "China Rare Disease Network." This meeting made many patients with rare diseases aware of the need to establish patient organizations, thereby facilitating the establishment of more rare disease patient organizations.

In 2013, the Chinese Organization for Rare Disorders (CORD), was formally established to create a platform, support the patient community, educate the public, research policy, and cooperate with international counterparts. The CORD has made important contributions to the development of Chinese rare disease patient organizations.

\section{Challenges faced by rare disease patient organizations in China}

Although rare disease patient organizations have developed for several years, there are still quite a number of problems:

Small number and dispersion of power There are currently about 80 rare disease patient organizations in China (10), but this number is far from enough compared to the number of rare diseases and patients. Although several preliminary networks of rare disease patient organizations have been created, these organizations remain weak due to different rates of development and a lack of strong cooperation or integration (11).

Low level of organizational specialization Most patient organizations have a low level of development and structure. The leader or head of the organization is a patient or family member. Most organizations are not registered. Most have no full-time staff, no fixed office, no consistent source of funding, and no standardized processes or systems.

Lack of organizational stability Almost all patient organizations are created and managed by patients and their families. Accordingly, the leader's health will greatly affect the organization's work and development. If a leader is ill or feels pressured by family, financial, or other reasons, the sustainability of the organization's operations will be affected.

Limited social influence and limited ability to access social resources Rare disease patient organizations are a new entrant among NGOs in China. Public concern is extremely limited. In addition, the public lacks sufficient understanding of rare diseases. As a result, social resources are neither allocated effectively nor efficiently, seriously impairing resource allocation.

\section{Suggestions}

Continued publicity The urgency of establishing rare disease patient organizations should be publicized. Public education on rare diseases should be continuously provided to attract attention from more groups.

Policy support The Government should actively provide more support at all levels to help patient organizations to develop. Patients and patient organizations should be empowered by certain policies, e.g. simplifying the registration process, increasing financial subsidies, and reducing taxes.

Team building The current members of patient organizations in China should receive more medical education as well as training in management skills to run those organizations more efficiently and sustainably. The quantity of members should be increased along with their quality. Organizations should recruit professionals with different backgrounds in medicine or NGO management to serve as external committees or consultants.

Communication and cooperation Organizations for patients with rare diseases should form alliances and build collaborative networks to the extent possible. This will help them to communicate and cooperate and will help expand educating the public, promoting policies, and securing resources. Patient organizations can also rely on some well-functioning charity organizations to conduct campaigns and secure resources. Patient organizations also need to further cooperate with research institutions and pharmaceutical companies to jointly promote research related to rare diseases. Moreover, communicating with foreign rare disease patient organizations is a way to join relevant collaborative networks and learn from their experience with organizational management. 


\section{Acknowledgements}

This study was supported by a grant from the Shanghai Municipal Health Commission for a Special Research Project related to Health Policies entitled "Study on the disease burden of and social security for the vulnerable population in Shanghai (Project number: 19Y04017)".

\section{References}

1. National Health Commission of the People's Republic of China and four other departments, Notice on the publication of the list of the first group of rare diseases. http://www.nhc.gov.cn/xxgk/pages/ viewdocument.jsp? dispatchDate $=$ \&staticUrl $=/ y z y g j /$ $s 7659 / 201806 / 393 a 9 a 37 f 39 c 4 b 458 d 6 e 830 f 40 a 4$ bb99.shtml\&wenhao $=($ accessed on April 5, 2019). (in Chinese)

2. He JJ, Kang Q, Hu JH, Song PP, Jin CL. China has officially released its first national list of rare diseases. Intractable Rare Dis Res. 2018; 7:145-147.

3. Rare disease information network, The China Rare Disease Drug Accessibility Report (2019) was solemnly released in Beijing. http://www.hanjianbing.org/sear ch!detail? id =8a9d475463869c1901693940728b04b2 (accessed on April 21, 2019). (in Chinese)

4. Biao W, Xiao YK, Chen R, Qin X, Ma Y, Hu Z. Study on social support for rare disease sufferers in China. Medicine and Society. 2012; 25:53-55. (in Chinese)

5. Liang TK. Social services for patients with rare diseases:
Real needs, main difficulties and countermeasures. Social Work and Management. 2016; 16:13-22. (in Chinese)

6. Souliotis K,Agapidaki E,Peppou LE, Tzavara C, Varvaras D, Buonomo OC, Debiais D, Hasurdjiev S, Sarkozy F. Assessing patient organization participation in health policy: A comparative study in France and Italy. Int J Health Policy Manag. 2017; 7:48-58.

7. Xiao L. Roles of patient organizations in rare disease research and orphan drug development. Journal of International Pharmaceutical Research. 2017; 44:209214. (in Chinese)

8. He JJ, Zhang YB, Xia SJ, Hu SL. The social security system for rare diseases in the European Union and its implications for China. Chinese Journal of Health Policy. 2012; 5:52-59. (in Chinese)

9. China-Dolls Center for Rare Disorders, Introduction to the China-Dolls Center for Rare Disorders. http:// chinadolls.org.cn/type/110 (accessed on May 12, 2019). (in Chinese)

10. Sohu net, China's first large-scale platform for communication among organizations of patients with different diseases, has been established. http://www. sohu.com/a/282884350_100116018 (accessed on May 12, 2019). (in Chinese)

11. Li DY, Zhan WY. Characteristics and dilemmas of the development of non-profit organizations for rare diseases in China. For All Health. 2013; 7:1-2. (in Chinese)

(Received April 13, 2019; Revised May 23, 2019; Accepted May 30, 2019) 